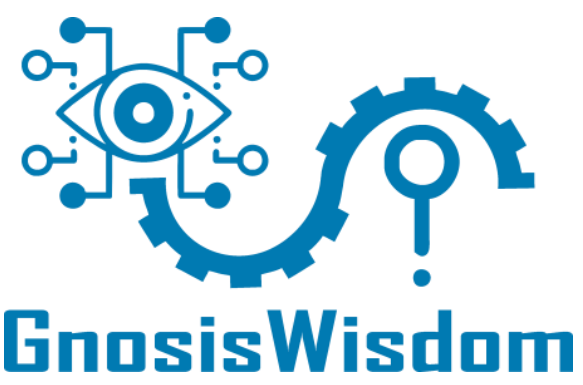

\title{
DIRECCIÓN ESTRATÉGICA PARA EL ORDENAMIENTO SOSTENIBLE DEL TRANSPORTE PÚBLICO EN LA PROVINCIAL DE TAYACAJA
}

\author{
TRATEGIC DIRECTION FOR THE SUSTAINABLE MANAGEMENT OF PUBLIC \\ TRANSPORTATION IN THE TAYACAJA PROVINCIAL
}

\author{
Autores \\ Papa Pio, Ascona Garcia \\ Universidad Nacional del Centro del Perú \\ Teofila, Chanca Mucha ORCID \\ Universidad Nacional de Huancavelica
}

\begin{abstract}
Resumen
La observación empírica es el deficiente ordenamiento del transporte público, considerada como el problema de la investigación; con el objetivo de demostrar la influencia de la dirección estratégica en el ordenamiento sostenible del transporte público, como hipótesis se consignó que la dirección estratégica influye significativamente en el ordenamiento sostenible del transporte público. La metodología utilizada de paradigma ontológico, el método científico deductivo, diseño experimental, tipo aplicado, nivel aplicativo, categoría de campo, enfoque cuantitativo y clase social, con población 7308 personas entre actores; contribuyentes y transportistas, muestreo probabilístico aleatorio, técnicas de recolección de datos la encuesta, instrumento cuestionario y la ficha, validada por expertos, con la confiabilidad de correlación igual a 0,94.
\end{abstract}

Palabras clave: Teoría pendiente desorden y felicidad.

\begin{abstract}
The empirical observation is the deficient arrangement of public transport, considered as the problem of the investigation; with the objective of demonstrating the influence of the strategic direction in the sustainable management of public transport, as a hypothesis it was stated that the strategic direction significantly influences the sustainable management of public transport.

The ontological paradigm methodology used, the deductive scientific method, experimental design, applied type, applicative level, field category, quantitative approach and social class, with a population of 7308 people among actors; taxpayers and carriers, random probability sampling, data collection techniques for the survey, questionnaire instrument and the form, validated by experts, with correlation reliability equal to 0.94 .
\end{abstract}

Keywords: Pending theory disorder and happiness. 


\section{GnasisWisdam}

\section{Introducción}

Afirmo que el servicio de transporte público de pasajeros y mercancías tiene la particularidad de ser muy desordenada, pésima en infraestructura, la tecnología es deplorable; las competencias administrativas y técnicas son insuficientes, el cumplimiento de la norma del transporte es defectuoso por falta de control serio, la cultura de traslado de los pasajeros es deficiente, la contaminación del medio ambiente es en escala ascendente, los incesante accidentes de tránsito con pérdida de vidas y materiales son permanentes. El principal interés de esta investigación, se concentra en evidenciar, demostrar y predecir progresivamente el problema con las experiencias y procesos del quehacer científico en el área de ciencias sociales; además en aportar con estrategias viables al ordenamiento de transporte público y disminuir los accidentes de tránsito.

Este caos del transporte público, es considerado muy común en el Perú. Para Valls (2011) un servicio público es "de acción de coordinación o conjunto de actividades jurídico administrativas y técnicas, que organiza el Estado o las corporaciones" (p. 441). Por lo tanto "el caos ocurre por los sistemas débiles no lineales, las burocracias tradicionales, el formalismo, las reglas imprecisas, la insatisfacción, la corrupción y leyes rígidas" (Cornejo, 2004, p. 9). Porque existe una desorganización de los actores, los ciudadanos sienten la desmoralización, advierten la incertidumbre muy a menudo, los contribuyentes y usuarios perciben intranquilidad, encuentran incongruencia en las acciones; también los actores directos califican esta situación como problema complejo "está en todas las áreas de las ciencias, cualquier variación, no importa su dimensión, el tipo, y la temporalidad en las condiciones originales de esos sistemas puede llegar a inducir alteraciones impredecibles" (Cárdenas \& Rivera, 2004, p. 139).

La posibilidad de la solución es la formulación, implementación y evaluación de la administración estratégica por ser "el arte y la ciencia de formular, implantar y evaluar las decisiones para que las organizaciones logren sus objetivos, con el propósito es explotar y crear oportunidades nuevas y diferentes para el futuro" (Fred \& Forest, 2017, p. 5). La acción para todo tipo de organización es el análisis de la situación actual como la existencia del caso, el análisis interno y externo denominado (FODA), la difusión de políticas sustanciales de la ecología ambiental con el ISO respectivo, extensión y uso de la herramienta LIENZO para la praxis de principios de calidad en el servicio público, la colaboración en la toma de decisiones y liderazgo, la marcha de sostenibilidad relacionado con el pensamiento cognitivo en el futuro, porque la sostenibilidad "es un paradigma general que abarca los problemas y temas ambientales, sociales, económicos y políticos que enfrentan las comunidades en todo el mundo" (UNESCO, 2012, pp. 19,48), es también "crear una estructura organizacional adecuada, instituir políticas, procesos y sistemas que apoyen la estrategia, motivar y recompensar a las personas e inculcar la disciplina para que se realicen las cosas" (Thompson, Peteraf, Gamble, \& Strickland, 2012, p. 323).

En tanto la presta del servicio de transporte público terrestre de personas o mercancías o mixta, se realiza a cambio de una retribución económica; es también la libertad de traslado de un punto a otro, a causa de la salud, la educación, el trabajo, la seguridad, el negocio en forma dinámica e integradora. Asimismo, 


\section{GnasisWisdam}

Guillamón \& Hoyos (2006) refieren que "la movilidad sostenible es un sistema de transporte que no perjudica a sus habitantes tampoco al ecosistema, más bien satisface la necesidad de desplazamiento de sus vecindarios" (p. 12). Por lo tanto, la investigación de desarrollo en cuatro capítulos, guiados por el siguiente flujo-grama.

\section{Figura 1}

Flujo-Grama de la Investigación

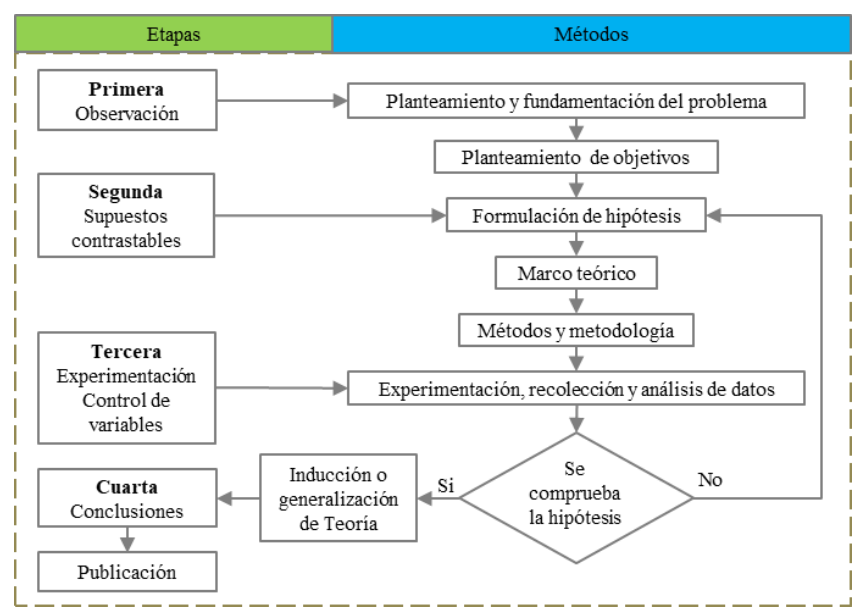

Nota. Etapas y métodos seguidos en la investigación.

Elaboración propia del investigador.

\section{Materiales y métodos}

Es de método científico Bunge (1987) porque "es un procedimiento para tratar un conjunto de problemas, o un rasgo característico de la ciencia tanto de la pura como de la aplicada, es decir cada clase de problemas requiere un conjunto de métodos o técnicas, reglas especiales" Ratifica Tamayo (2003) que el método científico como "un conjunto de procedimientos por los cuales se plantean los problemas científicos y se ponen a prueba las hipótesis y los instrumentos de trabajo investigativo". Mientras universalmente es deductivo porque parte de lo particular a lo general, es decir, está asociado frecuentemente con la investigación cuantitativa lo menciona (Behar Rivero, 2008). El tipo de investigación es aplicada porque resuelve los problemas prácticos inmediatos en orden de transformar los contextos actuales, así menciona Calderón \& Piñeiro (2003) $\mathrm{y}$ es de nivel aplicativo porque plantea resolver el problema natural apoyado en la innovación Supo (2012) y ratificado por Espinoza (2015), así mismo es predictiva porque prever situaciones futuras a partir de estudios absolutos con el estudio de probabilidades para identificar y analizar alternativas futuras, así refiere Hurtado de Barrera (2012). Sobre el enfoque es cuantitativo porque (Briones, 2002) menciona que el enfoque cuantitativo buscará la medición de los fenómenos sociales, mediante las variables, y utilizando las técnicas estadísticas. O sea que "recoge información empírica de objetos o características del fenómeno que pueden ser contar, pesar o medir, y arroja números como resultado" (Behar Rivero, 2008, pág. 28). Al igual que Montoya Zuluaga, y otros (2018) indican que el enfoque cuantitativo es la selección de contexto, estrategias y técnicas y que garanticen la generalización de los resultados. Según el diseño es experimental porque se ha manipulado deliberadamente la variable independiente (dirección estratégica) como causa para determinar los efectos de la variable dependiente (ordenamiento sostenible del transporte público), con un esquema lógico para 


\section{GnasisWisdam}

alcanzar los objetivos Pino (2013), en ese sentido también sostenida por Hernandez, Fernandez \& Baptitas (2014).

Tabla 1

Diseño experimental.

\begin{tabular}{cccc}
$\begin{array}{c}\text { Grupo } \\
\text { experimental } \\
\text { (asignado al azar) }\end{array}$ & $\begin{array}{c}\text { Pre } \\
\text { prueba }\end{array}$ & Tratamiento & $\begin{array}{c}\text { Post } \\
\text { prueba }\end{array}$ \\
\hline $\mathrm{G}_{\mathrm{E} 1}$ & $\mathrm{O}_{1}$ & $\mathrm{X}_{1}$ & $\mathrm{O}_{2}$ \\
\hline $\mathrm{GE}_{2}$ & $\mathrm{O}_{3}$ & $\mathrm{X}_{2}$ & $\mathrm{O}_{4}$ \\
\hline $\mathrm{G}_{\mathrm{C}}$ & $\mathrm{O}_{5}$ & -- & $\mathrm{O}_{6}$ \\
\hline
\end{tabular}

Nota: Elaboración propia del investigador, donde GE1 es el grupo experimental al azar de conductores y trabajadores de vehículos menores y mayores; GE2 es el grupo experimental al azar de autoridades, funcionarios, trabajadores $\mathrm{y}$ servidores públicos; GC es el grupo de control al azar de Contribuyentes o usuarios; $\mathrm{X} 1$ es el Tratamiento (Capacitación en seguridad vial, formalización y calidad de servicio), X2 es el Tratamiento (Plan de innovación del transporte público).

Sobre la categoría son exhaustivas, mutuamente excluyentes e independientes y discutibles, Cazau (2006) precisa que cada categoría de la variable independiente suele denominársela tratamiento experimental, en las variables cuantitativas, los porcentajes deben ser empleados como indicadores de calidad, por ejemplo, en la prestación del servicio son: la capacidad de respuesta (tiempo), seguridad, fiabilidad, empatía (confort, económico), elementos tangibles (infraestructuras), ecología ambiental.

Tabla 2

Técnica es muestreo de la investigación probabilístico.

\begin{tabular}{|c|c|c|c|c|}
\hline Estrato & Identificación & $\begin{array}{c}\mathbf{N}^{\mathbf{}} \\
\text { sujetos } \\
\text { en el } \\
\text { estrato }\end{array}$ & $\begin{array}{c}\text { Proporción } \\
(\mathbf{P})=\mathbf{n} / \mathbf{N}\end{array}$ & $\begin{array}{c}\text { Muestra } \\
\text { del } \\
\text { estrato }\end{array}$ \\
\hline 1 & $\begin{array}{c}\text { Contribuyentes } \\
\text { o usuarios }\end{array}$ & 5255 & $71.9 \%$ & 262 \\
\hline 2 & $\begin{array}{l}\text { Conductores y } \\
\text { trabajadores de } \\
\text { vehículos } \\
\text { menores y } \\
\text { mayores }\end{array}$ & 911 & $12.5 \%$ & 46 \\
\hline 3 & $\begin{array}{l}\text { Autoridades, } \\
\text { funcionarios, } \\
\text { trabajadores y } \\
\text { servidores } \\
\text { públicos }\end{array}$ & 1142 & $15.6 \%$ & 57 \\
\hline & & orrect & $100.0 \%$ & 365 \\
\hline
\end{tabular}

Nota: Elaboración propia del investigador, donde $\mathrm{n}$ en el tamaño de muestra igual a $365 \mathrm{~N}$ es la población igual a 7308 y P la proporción que es igual a la división de muestra entre población equivalente a 0,0522 .

Para Corbatta (2007) la fiabilidad es también la estabilidad en el momento de la observación, pero Kerlinger \& Lee (1999) mencionan que la confiablidad es la proporción de la varianza verdadera respecto a la varianza obtenida de los datos producidos por un instrumento de medición, donde el índice uno indica una confiablidad perfecta, asimismo Monje (2011) destaca que la confiabilidad se refiere a la capacidad del 


\section{GnasisWisdom}

instrumento para arrojar datos o mediciones que correspondan a la realidad que se pretende conocer. Mientras para Berna (2010) el análisis sirve para medir la fuerza o el grado de correlación entre las variables y objeto de estudio se aplica la formula estadística del Coeficiente de correlación de Pearson (r).

Tabla 3

Resumen de datos estadística descriptiva de la investigación.

\begin{tabular}{|c|c|c|c|c|c|c|}
\hline \multirow{2}{*}{ Sujetos } & \multirow{2}{*}{\multicolumn{2}{|c|}{ Prametros }} & \multicolumn{2}{|c|}{ Observacion 01} & \multicolumn{2}{|c|}{ Observacion $\mathrm{O2}$} \\
\hline & & & Estadístico & Error estándar & Estadístico & Error estándar \\
\hline \multirow{9}{*}{$\begin{array}{c}\text { Actores } \\
\text { (autoridades, } \\
\text { funcionarios, } \\
\text { trabajadores, } \\
\text { obreros y } \\
\text { servidores) }\end{array}$} & Media $=X$ & & 53.00 & 3.572 & 70.81 & 3.720 \\
\hline & $95 \%$ de intervalo de & Límite inferior & 45.84 & & 63.35 & \\
\hline & confianza para la media & Límite superior & 60.16 & & 78.26 & \\
\hline & Media recortada al 5\% & & 51.50 & & 70.67 & \\
\hline & Mediana $=\mathrm{Me}$ & & 49.00 & & 72.00 & \\
\hline & Varianza $=\mathrm{s} 2$ & & 727.464 & & 788.873 & \\
\hline & Des viación estándar $=\mathrm{S}$ & & 26.972 & & 28.087 & \\
\hline & Asimetría = As & & .571 & .316 & .089 & .316 \\
\hline & Curtosis & & -.816 & .623 & -.810 & .623 \\
\hline \multirow{9}{*}{$\begin{array}{c}\text { Contribuyentes y/ } \\
\text { usuarios }\end{array}$} & Media $=X$ & & 48.26 & 1.403 & 70.72 & 1.740 \\
\hline & $95 \%$ de intervalo de & Límite inferior & 45.50 & & 67.29 & \\
\hline & confianza para la media & Límite superior & 51.03 & & 74.14 & \\
\hline & Media recortada al 5\% & & 46.62 & & 70.58 & \\
\hline & Mediana $=\mathrm{Me}$ & & 48.00 & & 72.00 & \\
\hline & Varianza $=\mathrm{s} 2$ & & 515.712 & & 793.644 & \\
\hline & Des viación estándar $=\mathrm{S}$ & & 22.709 & & 28.172 & \\
\hline & Asimetría $=$ As & & .721 & .150 & -.030 & .150 \\
\hline & Curtosis & & -.172 & .300 & -.870 & .300 \\
\hline \multirow{9}{*}{$\begin{array}{c}\text { Transportistas } \\
\text { (empresarios, } \\
\text { conductores de } \\
\text { vehículos mayores } \\
\text { - menores y } \\
\text { trabajadores) }\end{array}$} & Media $=X$ & & 53.02 & 3.276 & 71.96 & 3.991 \\
\hline & $95 \%$ de intervalo de & Límite inferior & 46.42 & & 63.92 & \\
\hline & confianza para la media & Límite superior & 59.62 & & 79.99 & \\
\hline & Media recortada al $5 \%$ & & 51.72 & & 71.91 & \\
\hline & Mediana $=\mathrm{Me}$ & & 48.00 & & 72.00 & \\
\hline & Varianza $=\mathrm{s} 2$ & & 493.533 & & 732.531 & \\
\hline & Des viación estándar $=\mathrm{S}$ & & 22.216 & & 27.065 & \\
\hline & Asimetría $=$ As & & .886 & .350 & .060 & .350 \\
\hline & Curtosis & & .036 & .688 & -.722 & .688 \\
\hline
\end{tabular}

Nota: Elaboración propia del investigador.

El análisis estadístico según las opiniones de los dos grupos experimentales y un grupo de control, antes del tratamiento y después del tratamiento se resumen en la siguiente tabla. 


\section{GnosisWisdam}

\section{Resultados}

A juicio de Santa \& Soraya (2016) el análisis de varianza de un experimento es para lograr el conocimiento acerca del estudio y requiere la comparación de comportamientos en los distintos niveles o tratamientos. Es decir, la primera observación obtenida de la muestra, la asimetría es positiva con alargamiento hacia la derecha, es decir que la Moda $\leq$ Mediana $\leq$ Media, y el valor calculado de asimetría $(\mathrm{As}=1.09)$ es decir es mayor que cero. Mientras en la segunda observación se tiene una asimetría negativa con oblicua mínima hacia la izquierda, es decir que la Media $\leq$ Mediana $\leq$ Moda, y el valor calculado de asimetría (As $=-0.04)$ es decir es menor que cero, lo que se respeta mencionado por De La Puente (2018), tal como se percibe que la gráfica de la derecha muestra una curva muy similar a una simetría según las condiciones de simetría y asimetría.

Figura 2

Análisis de determinación de simetría de la investigación.

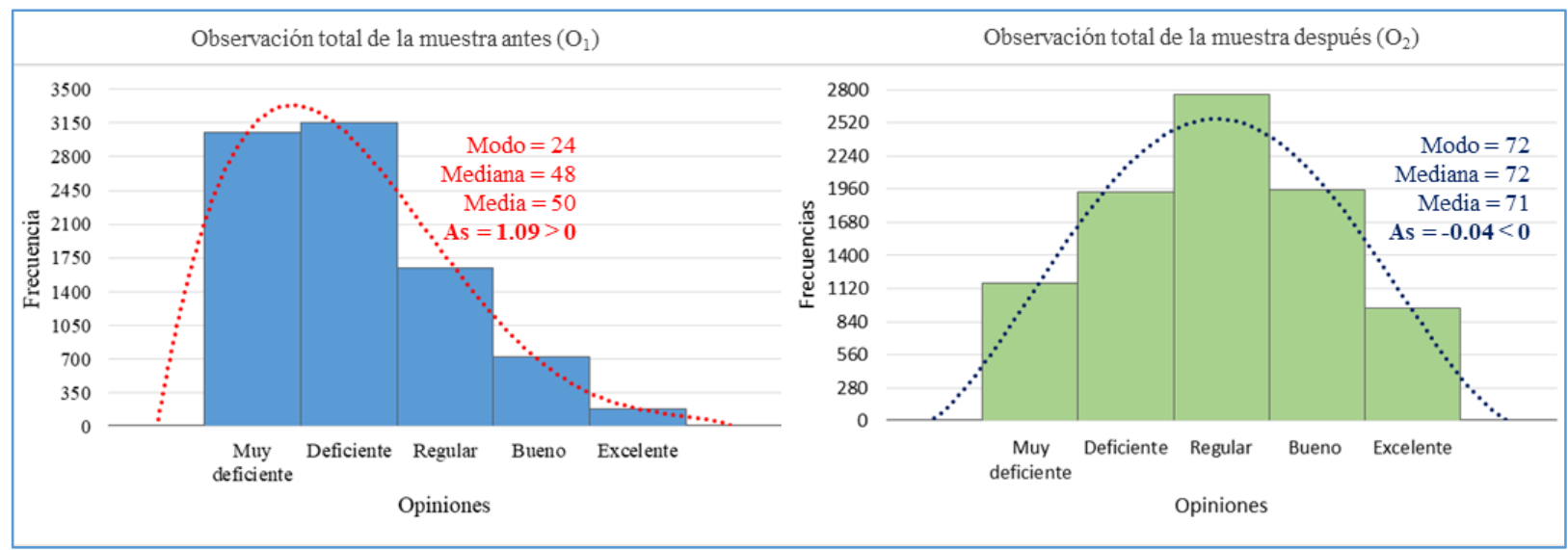

Fuente: Elaboración propia del investigador.

Los datos provienen de muestras aleatoria paramétrica y analizado la normalidad de la curva de distribución resulta el p-Valor igual a $(0,000$ y 0,000) de mediciones antes y después respectivamente, realizando la comparación con el valor Alfa $(\alpha)$ igual a 0,05 , es claro que las mediciones como antes y después es menor que $\alpha$, entonces la distribución no es normal, asimismo la prueba hipótesis con el estadístico de puntuación
Z es igual 1,29 entonces se acepta la hipótesis nula (Ho), y se rechaza la hipótesis alterna (H1), es decir, la dirección estratégica influye significativamente en el ordenamiento sostenible del transporte público, después de aplicar los tratamientos $(\mathrm{X} 1+\mathrm{X} 2)$, corroborado con las modas de ambas mediciones $(\mathrm{O} 2=72)$ y $(\mathrm{O} 1=24)$. Asimismo, en la siguiente grafica de cajas se observa la diferencia en las barras acumuladas de 


\section{GnasisWisdam}

los grupos experimentales y control. Las barreas de caja de la O1 están muy a al inferior de recta principal, en tanto las barras de la O2. están aproximadamente al centro del plano cartesiano, es decir, los valores de medias en la segunda medición están ala medio que coinciden con las respuestas de opiniones (mayor igual a regular).

Figura 3

Gráfica de caja de la distribución de datos de los tres grupos.
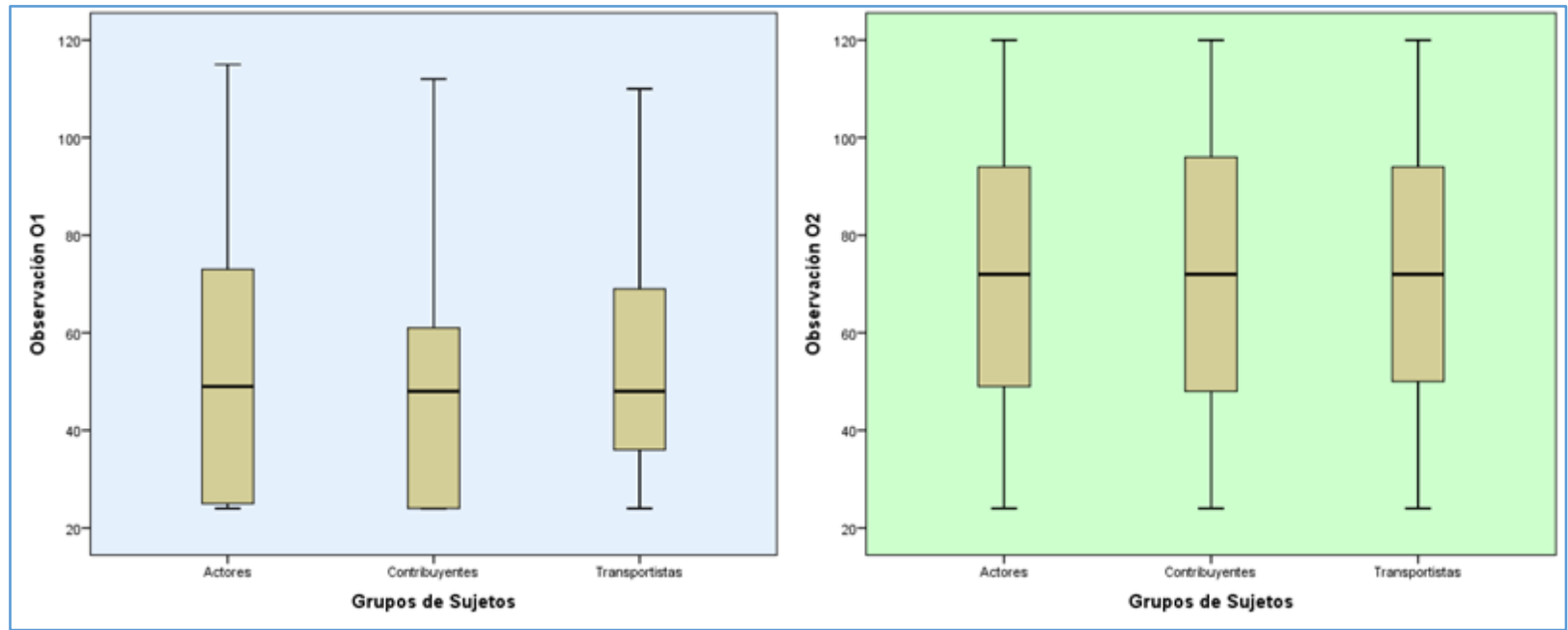

Fuente: Elaboración propia del investigador.

\section{Figura 5}

Predicción de opiniones de los tres grupos.

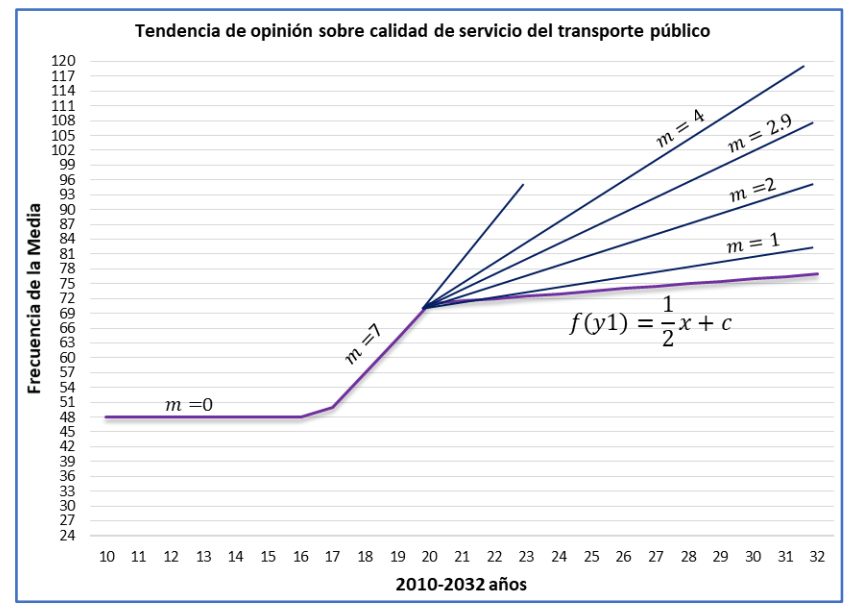

Fuente: Elaboración propia del investigador, donde $\mathrm{f}(\mathrm{y})$ = función de la recta lineal, $\mathrm{m}=$ pendiente de la recta.
La administración pública de servicios es lineal porque tiene relación directa con la función de la recta con tendencia creciente o decreciente. A pesar de ser complejo es necesario la toma de decisiones oportunas como funcionales $y$ estratégicas. Al extremo de repetir los mismos estímulos o tratamientos a lo largo de mucho tiempo, esto puede terminar convirtiéndose en una costumbre rutinaria. $\mathrm{O}$ sea, cuando la pendiente $\mathrm{m}$ es igual a cero, la recta se mantiene en la posesión horizontal, este resultado es muy posible en la realidad significando que no se ejecutan ningún tratamiento o estímulo complementario de lo que se realizó con anterioridad, pero no tiene la 


\section{GnasisWisdam}

sostenibilidad porque pequeños cambios en la sociedad pueden variar negativamente el sentido del objetivo, es decir, no es recomendable mantener. Pero cuando la pendiente $\mathrm{m}$ es igual a un medio, significa que esta orientación de la recta es creciente, sostenible y posible de obtener en la realidad, al ejecutar tratamiento o estímulo complementario a lo que ya se efectuó. Ahora bien, cuando la pendiente $\mathrm{m}$ es igual a uno, significa que esta orientación de la recta es positiva, sostenible y posible de obtener en un escenario determinado, con la ejecución de tratamiento o estímulo planificados a largo plazo, aunque no es recomendable para objetivos promisorios sino para resultados pausados en el futuro. Pero cuando la pendiente $\mathrm{m}$ es igual a dos, significa una administración lineal creciente en el plano, también es posible obtener en el realismo social, con la ejecución de tratamientos o estímulos planificados en función del objetivo social, ello no es recomendable para satisfacer las necesidades del contribuyente, en forma inmediata, pero si para largo plazo. Sin embargo, cuando la pendiente $\mathrm{m}$ es igual a 2,9 , siendo este valor como máximo de la función $(\mathrm{y}=\mathrm{mx}+\mathrm{c})$ para un tiempo de 12 años, la tendencia de la recta es similar a $\mathrm{m}$ es igual a dos. Pero cuando la pendiente $\mathrm{m}$ es igual o mayor a cuatro, significa una administración lineal creciente en el plano, también es posible obtener en la realidad social, con la ejecución de tratamientos o estímulos planificados a un determinado grupo social en función del objetivo organizacionales y sociales, ello es recomendable para cumplir objetivos atrevidos doblegando esfuerzos de recursos humanos, ecónomos y ambientales, sin garantiza la sostenibilidad, por ser muy riesgoso y con consecuencias costosos.

\section{Discusión}

Monje (2011) menciona que "la prueba deber estar seguida de una discusión sobre las ambigüedades o dificultades presentadas en el transcurso del proceso de investigación, no limitarse a un grupo de personas con un enfoque de la teoría fundamental" (p. 166). Por su parte Hurtado de Barrera (2012) refiere al respecto y sin dejar de citar a Espinoza (2015) refiere sobre la discusión de la investigación.

\section{Discusión en función de resultado}

Los datos provienen del muestreo aleatoria paramétrica, del análisis de normalidad en la curva de distribución, del resultante del p-Valor igual a $(0,000$ y 0,000$)$ de mediciones antes y después respectivamente, realizando la comparación con el valor Alfa $(\alpha)$ igual a 0,05 , es claro que las mediciones como antes y después es menor que $\alpha$, entonces la distribución no es normal, asimismo la prueba hipótesis con el estadístico de puntuación $\mathrm{Z}$ es igual 1,29 entonces se acepta la hipótesis nula (Ho), y se rechaza la hipótesis alterna (H1), es decir, que la dirección estratégica influye significativamente en el ordenamiento sostenible 


\section{GnasisWisdam}

del transporte público, después de emplear los tratamientos $(\mathrm{X} 1+\mathrm{X} 2)$, corroborado con las modas de ambas mediciones $(\mathrm{O} 2=72)$ y $(\mathrm{O} 1=24)$. Como se ha planteado en las hipótesis.

\section{Discusión en función de las teorías}

En esta investigación se detalla minuciosamente las teorías específicas que justifican la existencia de las variables independiente y dependiente. De estos resalto sus principios, sus enfoques, sus paradigmas, sus alcances, sus dimensiones y lo más importante su conceptualización referidos por los autores, Sin duda me es necesario enfatizar la teoría de la toma de decisiones como el proceso de aprendizaje y escoger una opción entre varias alternativas en base a los objetivos, preferencias, estrategias, resultados estadísticos de situaciones similares, la toma de decisiones individuales y colectivas en circunstancias de incertidumbre teoría de caos, y considero coherente la posición de Amaru (2009) que la actitud en el futuro es ser proactivo. También a Drucker, Hesselbein \& Snyder (2015) quien menciona que cada organización social existe con el fin de conseguir una mejora valiosa en la vida de las personas, su propósito y su razón de ser es conseguir la mejora ansiada.

\section{Conclusiones}

Generalizando se demuestra que la dirección estratégica, influye significativamente en el ordenamiento sostenible del transporte público, es decir, al aplicar el tratamiento X1 acción de capacitación a los transportistas, el tratamiento X2 ejecución del plan de innovación a los actores y al no emplear ningún tratamiento a los contribuyentes y usuarioa, se aceptar la Hipótesis alternativa (H1), porque las mediciones antes resultan que la media poblacional es igual a 50, la mediana antes igual a 48, la moda antes igual 24 $\mathrm{x}_{-}^{-} \mathrm{a}=50, \llbracket \mathrm{Me} \rrbracket \_\mathrm{a}=48, \llbracket \mathrm{Mo} \rrbracket \_\mathrm{a}=24$, ahora bien al realizar las mediciones después resultan que la media poblacional es igual a 71, la mediana después es igual a 72, la moda después es igual a $72 \mathrm{x}{ }_{-}^{-} \mathrm{d}=71, \llbracket \mathrm{Me} \rrbracket \_\mathrm{d}=72$, 『Mo】_d=72, en tanto los datos de las medias poblacionales proviene de una muestra paramétrica, asumiendo el valor de la significancia igual de cinco por ciento, justificado con el cálculo del estadística de prueba de puntuación $\mathrm{Z}$ después se ubican dentro de un rango bajo la curva de Gauss, denominado como área o zona de aceptación y pertenecen a números reales enteros, estos son valores la media población $\mu$ es igual a $68.02 \leq \mu<73.76$, afirmado con la normalidad del calculo de p-valor $(0,000 \mathrm{y}$ 0,000) medición antes y después respectivamente, esto significa que 0,000 es menor que 0,05 valor de alfa, siendo los datos (promedio de las medias) de la variable independiente tanto antes y después, no proviene de una distribución normal, y ratificado con el cálculo del coeficiente de correlación de Pearson es rxy igual a 0,94 donde la confiabilidad es muy fuerte. En resumen, influye significativamente peno no lo suficiente. 


\section{GnasisWisdam}

\section{Referencias}

Amaru Maximiano, A. C. (2009). Fundamentos de administración: teoría general y proceso administrativo (Primera ed.). (P. M. Rosas, Ed., \& J. L. Servin, Trad.) Pearson Educación de México, S.A.

Behar Rivero, D. S. (2008). Introducción a la Metodología de la Investigación (Primera ed.). Lima: Shalom.

Bernal Torres, C. A. (2010). Metodología de la investigación administración, economía, humanidades y ciencias sociales (Tercera ed.). Mexico: Pearson Educación de Colombia LTDA.

Briones, G. (2002). Epistemología de las ciencias sociales. (D. B. Maldonado, Trad.) Bogota, Colombia: ARFO Editores e Impresores Ltda.

Bunge, M. (1987). La investigación científica (Segunda ed.). México: Ariel S.A.

Calderón Fornaris, P. A., \& Piñeiro Suárez, N. (2003). Metodología de la investigación científica. Habana: PRENTICE HALL.

Cazau , P. (2006). Introducción a la investigación en ciencias sociales (Tercera ed.). Buenos Aires: Universidad del Museo Social Argentino.

Corbatta, P. (2007). Metodología y técnicas de investigación social (Primera ed.). España: McGraw/Hill-Interamerica de España.

Cornejo Álvarez, A. (2004). Complejidad y caos (Primera ed.). (M. Proust, Ed.) España: DR@ 2004.

De La Puente Viedna, C. (2018). Estadística descriptiva e inferencial (Primera ed.). Madrid: IDT CB. Madrid - Spain - Europe.
Drucker, P. F., Hesselbein, F., \& Snyder Kuhl, J. (2015). Las 5 claves de Peter Drucker. Profit Editorial.

Espinoza Montes, F. (2015). La tesis universitaria (Primera ed.). Huancayo: Master SAC.

Fred R., D., \& Forest R., D. (2017). Conceptos de administración estratégica (Decimoquinta ed.). (E. M. Hernan, Trad.) Mexico: Pearson Educacion.

Guillamón, D., \& Hoyos , D. (30 de Noviembre de 2006). Movilidad Sostenible de la teoría a la práctica.

1-49. Zaindu gure etxea. Obtenido de http://www.bantaba.ehu.es

Hernandez Sampieri, R., Fernandez Collado, C., \& Baptitas Lucio, M. (2014). Metodologia de la Investigacion (Sexta ed.). Mexico: McGrawHILL/Interamericana Editores S.A.

Hurtado de Barrera, J. (2012). Metodología de la investigación (Cuarta ed.). Bogota Caracas: Guirón Edicione.

Kerlinger, F. N., \& Lee, H. D. (1999). Investigación del comportamiento (Cuarta ed.). (L. E. Ayala, Trad.) Chile: McGraw-Hill Interamericana.

Kondo, M. (2016). La felicidad después del orden. (V. C. Díez, Trad.) Tokyo: Penguin Random House Grup o Editorial S.A.U. Obtenido de http://www.megustaleer.com

Molinero Molinero, Á. R., \& Sánchez Arellano, L. I. (2005). Transporte público: planificación, diseño, operación y administración (Segunda ed.). Mexico: Universidad Autonoma del Estado de Mexico. 


\section{GnasisWisdam}

Monje Álvarez , C. A. (2011). Metodología de la investigación cuantitativa y cualitativa. Neiva: Universidad surcolombiana.

Montoya Zuluaga, P., Cogollo Ospina, S., Duran Palacio, N., Castaño Torres, S., Calderón Vallejo, G. A., Calle Piedrahita, J. S., \& García Chacon, B. E. (2018). Situaciones y retos de la investigación en Latinoamérica (Primera ed.). Colombia: Universidad Catolica Luis Amigo.

Pino Gotuzzo, R. (2013). Metodologia de la investigacion (segunda reimpresion ed.). (S. Marcos, Ed.) Lima, Lima, Peru: San Marcos E.I.R.L.

Porter, M. E. (2008). Estrategia competitiva (Trigésima octava ed.). (J. E. Callejas, Ed.) Mexico: Grupo Patria CulturalL S.A. de C.V.

Salinas Meruane, P., \& Cardenas Castro, M. (2009). Métodos de investigación social (Segunda ed.). Quito: Universidad Catolica del Norte.

Santa María, C. R., \& Soraya Buccino , C. (2016). Elementos de Probabilidad y Estadistica (Primera ed.). (U. N. Mareno, Ed.) Argentina: UNM Editora.

Supo, J. (2012). Seminarios de investigación científica sinopsis del libro 2012. sinopsis : seminariosdeinvestigacion. Obtenido de http://seminariosdeinvestigacion.com/sinopsis

Tamayo Tamayo, M. (2003). El proceso de investigación científica (Cuarta ed.). México : LIMUSA S.A.

Thompson, A. A., Gamble, J. E., Peteraf, M. A., \& Strickland, I. A. (2012). Administración estratégica (Vol. 18). Mexico: McGRAWHILL/INTERAMERICANA.
Torres, Z. (2014). Administración estratégica (Primera ed.). México: Grupo Editorial Patria S.A.

Valls Hernández, S. (2011). La noción del servicio público en el estado social de derecho. En S. Valls Hernández, \& C. Matute Gonzales, Nuevo Derecho Administrativo (Primera ed., págs. 405-422). Purrua SA. Obtenido de https://es.calameo.com/read/00057984990b61e020f82 Velásquez M., C. V. (2015). Sistemas Integrados de Transporte Masivo (SITM). (Tesis Doctoral). Universidad de Barcelona. Obtenido de http://diposit.ub.edu/dspace/handle/2445/67821 